\title{
Geometrical pinning of magnetic vortices induced by a deficit angle on a surface: Anisotropic spins on a conic space background
}

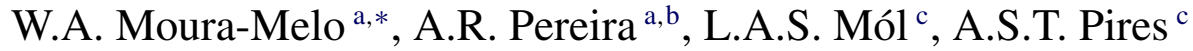 \\ ${ }^{a}$ Departamento de Física, Universidade Federal de Viçosa, 36570-000, Viçosa, Minas Gerais, Brazil \\ ${ }^{\mathrm{b}}$ Physics Department, University of Bolonga, Via Irnerio 46, I-41126, Bolonga, Italy

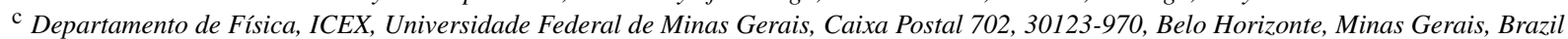

Received 26 June 2006; received in revised form 4 July 2006; accepted 5 July 2006

Available online 14 July 2006

Communicated by V.M. Agranovich

\begin{abstract}
We study magnetic vortex-like excitations lying on a conic space background. Two types of them are obtained. Their energies appear to be linearly dependent on the conical aperture parameter, besides of being logarithmically divergent with the sample size. In addition, we realize a geometrical-like pinning of the vortex, say, it is energetically favorable for it to nucleate around the conical apex. We also study the problem of two vortices on the cone and obtain an interesting effect on such a geometry: excitations of the same charge, then repealing each other, may nucleate around the apex for suitable cone apertures. We also pay attention to the problem of the vortex pair and how its dissociation temperature depends upon conical geometry.
\end{abstract}

(c) 2006 Elsevier B.V. All rights reserved.

\section{Introduction and motivation}

Non-linear excitations play important role for understanding several properties of physical systems. For instance, a number of phase transitions observed in cosmology, high energy, and condensed matter physics are accompanied by such kind of excitations. Particularly, in low dimensional magnetic materials, such excitations develop important roles. This is the case for two-dimensional spin models with planar symmetry, where a topological phase transition takes place due to vortex-antivortex dissociation [1,2]. Actually, such a transition (Berezinskii-Kosterlitz-Thouless transition) is expected to occur in a variety of systems such as films of superfluid helium, superconducting materials, Josephson-junctions as well as certain magnetic, gaseous and liquid-crystal systems. Whether a similar scenario would take place in other surface backgrounds (say, curved support) remains still an open question. Of course,

\footnotetext{
* Corresponding author.

E-mail addresses: winder@ufv.br (W.A. Moura-Melo), apereira@ufv.br (A.R. Pereira), lucasmol@ fisica.ufmg.br (L.A.S. Mól), antpires@fisica.ufmg.br (A.S.T. Pires).
}

a first step in this direction is to know how vortices behave in non-Euclidean spaces. Concerning this, we should emphasize that magnetic materials structured in curved geometry have attracted a great deal of efforts in the last years. Such an interest partially lies on the fact that magnetic samples can be currently manipulated at very small scales (around some dozens of nanometers or even smaller) and/or several shapes [3]. The knowledge of their magnetic properties is important for many potential applications, like devices for data recording and logic gates [4]. Therefore, a number of works has been devoted to study curved magnetic surfaces like cylinders, spheres, cones, and so forth. Several results that emerge from the interplay between magnetic and geometric effects have been obtained. For example, on the surface of a magnetic cylinder, solitons appear to be sine-Gordon-like excitations [5], which tend to deform the cylindrical surface in order to relieve the geometrical frustration brought about by nonconstant curvature, anisotropies, Zeeman effect, etc. [6]. Now, on a conical support their energy gets lower as long as cone is narrowed, indicating that such surfaces could be thought as pinning defects for solitons [7] (see, however [8]). In addition, transition from flower to vortex-like magnetization has been experimentally observed in ferromag- 


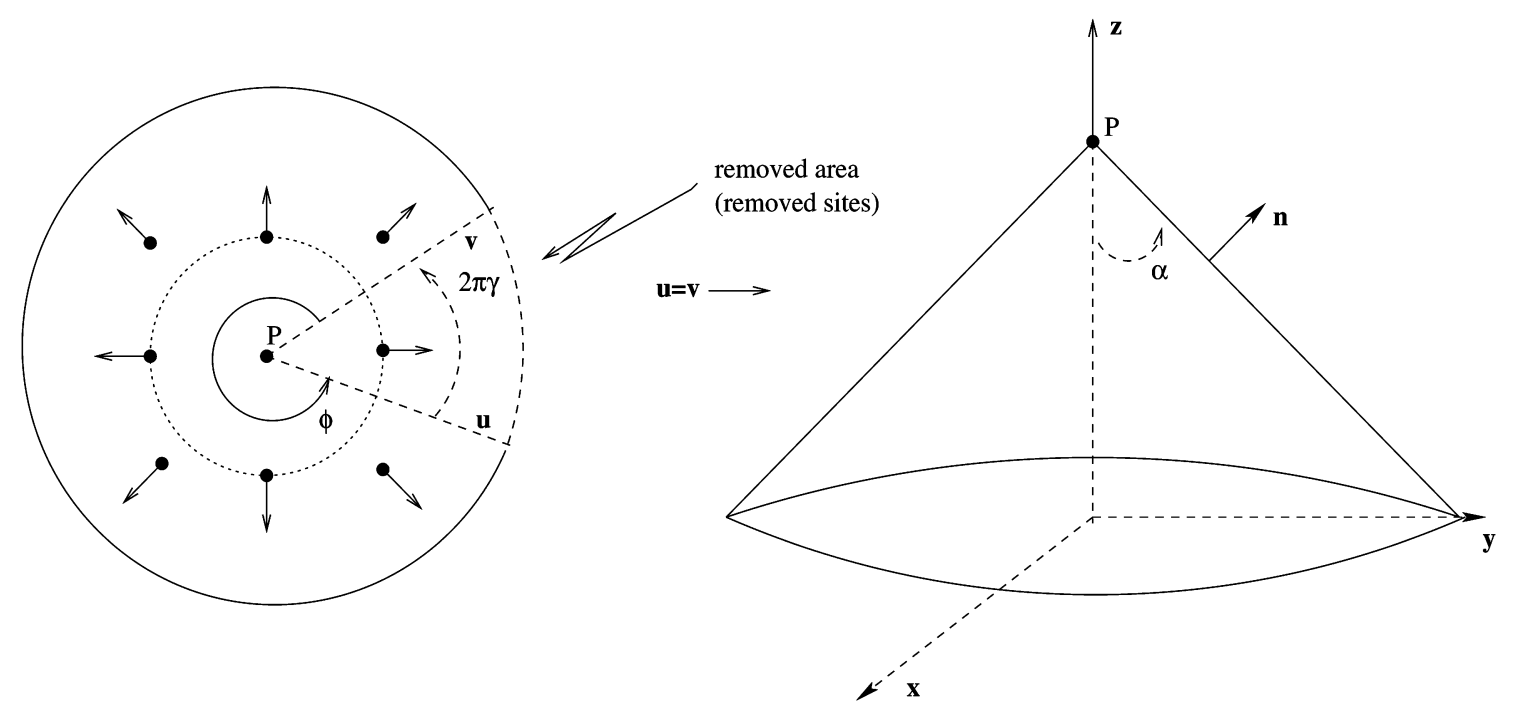

Fig. 1. Here, it is shown cut and removal of a wedge-type area equal to $2 \pi \gamma r^{2}$ from the original disc area, $2 \pi r^{2}$. Removing magnetic sites, now as $\phi$ goes from 0 to $\pm 2 \pi(1-\gamma)= \pm 2 \pi \beta$ the identification of lines $\mathbf{u}$ and $\mathbf{v}$ geometrically forces the spins to take a complete round. Note that $\beta=\sin (\alpha) \in(0,1)$, for usual cones. (For improving visualization, arrows representing spins are not depicted on the cone.)

netic nanosized cones $[9,10]$. Therefore, investigation of the basic properties of such excitations in conical support is important not only from a fundamental point of view, it is also appealing from applied physical grounds. Moreover, it should be emphasized that even 'more exotic' manifolds, like those with negative curvature (hyperbolic spaces) have been quite recently studied in connection with condensed matter and statistical physics [11].

Here, our attention is focused on vortex-like excitations lying on a circular conical surface covered by an anisotropic classical Heisenberg magnet. Our initial motivation for considering such a problem was to analyze how lattice defects (here a deficit angle) affect excitations whose solution is angle-like dependent. Such spatial defect is particularly interesting because it makes the space locally Euclidean while it presents nontrivial global curvature effects (due to the boundary conditions which identify opposite points of the wedge cut out of space, as shown in Fig. 1). Indeed, conic space can be described by a spatial metric with a Riemann-Christoffel curvature tensor which vanishes everywhere, except in one point, where there is a conical singularity characterized by a deficit angle [12]. In a planar magnetic material, such a (area) deficit could be artificially built without cutting out any part of the space, rather it could be obtained substituting the magnetic atoms by nonmagnetic sites in the large region covered by the deficit angle. In addition, the spins placed at opposite sites of the wedge should be identified. While this complex construction should be a very difficult task nowadays (mainly because of the nontrivial boundary conditions), this physical system could be exactly reproduced in a simpler manner: a conical support with appropriate magnetic coating. We shall see in what follows that, by virtue of the deficit angle, vortex excitations present lower energy than their counterpart on a standard flat plane. Actually, energy diverges logarithmically as sample conic area blows up, but decreases linearly as cone aperture angle, $2 \alpha$, is lowered. Here, we distinguish between two types of vortices: in-cone and out-of-cone. The main difference between them lies in the fact that the latter has a core where spins present components out of the surface. We also relate the appearance of each type with conical aperture and location on the cone. Such issues will be the subject of Section 2. Results from this section open the possibility of a geometrical controlling of the vortex-like excitations energy on actual finite-size samples and of pinning them around conically shaped structures. Indeed, we shall realize that the magnetic vortex tends to nucleate around the conical apex (Section 3).

We also address the problem of two vortices on the conical magnet. This shall be done in Section 4. Similarly to the usual flat plane case, a vortex pair on the conical surface presents finite energy, whose value appears to increase logarithmically with the separation of the vortices while decreasing linearly as the cone is narrowed. An interesting result emerging from this section is the possibility of vortices with the same charge, then mutually repealing, of nucleating around the apex. This is carried out for two distinct arrangements of the excitations and for both cases we determine the suitable conical apertures supporting the nucleation. Following, we analyze possible consequences of the curvature on the problem of depairing of vortices, namely, how critical temperature is sensitive to geometrical parameters. The conical surface is, perhaps, the first step in this direction since it is locally Euclidean and, therefore, its geometry keeps some similarities with the standard and well studied flat plane. Finally, we close this work by pointing out our conclusions and prospects for future investigation.

\section{The model and vortex-like solutions}

Let us consider an anisotropic Heisenberg-like model for nearest-neighbors interacting spins on a two-dimensional lattice, like below:

$H_{1}=-\frac{J}{2} \sum_{\langle i, j\rangle} \mathcal{H}_{i, j}=-\frac{J}{2} \sum_{\langle i, j\rangle}\left(S_{i}^{x} S_{j}^{x}+S_{i}^{y} S_{j}^{y}+\lambda S_{i}^{z} S_{j}^{z}\right)$, 
where $J>0$ is the ferromagnetic exchange coupling between neighbor spins and $\vec{S}_{i}=\left(S_{i}^{x}, S_{i}^{y}, S_{i}^{z}\right)$ is the spin operator at site $i$. Parameter $\lambda$ answers for the anisotropy between spin couplings: for $\lambda>1$ spins tend to align along the $z$-axis (easyaxis regime); for $\lambda=1$ we have the isotropic case; while for $0<\lambda<1$ one gets the easy-plane regime. Finally, $\lambda=0$ leads to the so-called $X Y$ model.

Whenever dealing with a general surface, we could also take into account another model which incorporates possible anisotropy due to the alignment of spins along the normal of this surface, like follows:

$H_{2}=-\frac{J}{2} \sum_{\langle i, j\rangle} \mathcal{H}_{i, j}+\frac{J \delta}{2} \sum_{i}\left(\vec{S}_{i} \cdot \hat{n}_{i}\right)^{2}$

where $\hat{n}_{i}$ is a unity vector normal to the surface at each site, $i$. Thus, for $\delta>0$ the spins tend to be locally parallel to the surface (we shall take $\delta \geqslant 0$, hereafter). This may be particularly important for taking into account, for instance, magnetostatic energy associated with topological excitations lying on a finite and nonplanar background. In fact, $\delta$-term works like a singleion anisotropy parameter in a general surface, so that as long as we are dealing with a flat one, say, $x y$-plane, then such a term recover the usual single-ion anisotropy, $\delta\left(S_{i}^{z}\right)^{2}$. Results concerning such an issue and vortex stability in (flat) square, hexagonal and triangular lattices are available in the works of Refs. $[13,14]$.

In the continuum approach of spatial and spin variables, which is valid at sufficiently low temperature and long-wavelength limit, Hamiltonian above may be expanded to yields

$$
\begin{aligned}
H & =E_{0}-\frac{J}{2} \iint_{\Omega} \mathcal{H}_{\text {cont }} d \Omega \\
& =E_{0}+\frac{J}{2} \iint_{\Omega}\left[(\mathbf{D} \vec{S})^{2}+\frac{\delta}{a^{2}}(\vec{S} \cdot \hat{n})^{2}\right] d \Omega,
\end{aligned}
$$

where $E_{0}$ is the ground state energy, $a$ is the lattice spacing parameter, and we have used, for simplicity $\lambda=1$. In turn, the classical spin state is taken to be $\vec{S}=(\sin \theta \cos \Phi, \sin \theta \sin \Phi$, $\cos \theta)$, with $\theta=\theta(\vec{x}, t)$ and $\Phi=\Phi(\vec{x}, t)$. Here, $\hat{n}$ is an unity vector field normal to the surface at each point. Therefore, the $S^{2}$-parametrization of $\vec{S}$ is such that, at each point of the physical surface, $\theta$ is the angle between $\vec{S}$ and $\hat{n}, \vec{S} \cdot \hat{n}=\cos (\theta) \equiv m$, while $\Phi$ is the local polar angle. In turn, the integral is taken over an arbitrary surface, $\Omega$, while $\mathbf{D}=\sum_{i} \hat{e}^{i} D_{i}$ represents the covariant derivative on such a space. Recalling that $\vec{S}^{2}=1$, the term $\iint_{\Omega}(\mathbf{D} \vec{S})^{2} d \Omega$ in Hamiltonian above is the nonlinear $\sigma$ model in a curved space background. Therefore, Hamiltonian (3) describes a kind of anisotropic nonlinear $\sigma$ model on a curved surface.

As it is well known, the main ingredient to obtain a vortex solution is a field equation that reduces in some limit to a Laplacian yielding the energy density $(\vec{\nabla} \Phi)^{2}$, where $\Phi$ is the phase. Such a limit is implicit in our system as one can easily see below. First, due to the local Euclidean nature of the conical surface, it is convenient to use local flat coordinates [7,12] defined as $\vec{\rho}=(\rho, \tau)=\left(r^{\beta} / \beta, \beta \phi\right)$, where $\vec{r}=(r, \phi)$ are as- sumed to be the usual polar coordinates. Thus we can rewrite Hamiltonian (3) as follows:

$$
\begin{aligned}
H= & \frac{J}{2} \int_{0}^{\infty} \int_{0}^{2 \pi \beta}\left[\frac{\left(\vec{\nabla}_{\vec{\rho}} m\right)^{2}}{1-m^{2}}+\left(1-m^{2}\right)\left(\vec{\nabla}_{\vec{\rho}} \Phi\right)^{2}\right. \\
& \left.+\frac{\delta}{a_{\rho}^{2 / \beta}} \beta^{-2} \rho^{\frac{2-2 \beta}{\beta}} m^{2}\right] \rho d \rho d \tau,
\end{aligned}
$$

where $\vec{\nabla}_{\vec{\rho}}$ is the gradient written in terms of coordinates $(\rho, \tau)$ and $a_{\rho}=a^{\beta} / \beta$ is the analog of the actual lattice spacing parameter $a$, written in $(\rho, \tau)$ coordinates. Note that a spin lying on this geometry experiences an effective anisotropy $\Delta=$ $\delta \beta^{-2} \rho^{\frac{2-2 \beta}{\beta}}$ that depends on its position (site) $\rho$ on the surface as well as the cone aperture, measured by the parameter $\beta=\sin (\alpha), \alpha$ being the cone half-angle (see Fig. 1 ).

The second step for getting the vortex solutions is to obtain the equations of motion on this support. Their static counterparts can be found from Eq. (4), and read like follows:

$$
\begin{aligned}
& \nabla_{\vec{\rho}}^{2} m+\frac{m\left(\vec{\nabla}_{\vec{\rho}} m\right)^{2}}{1-m^{2}}+m\left(1-m^{2}\right)\left[\left(\vec{\nabla}_{\vec{\rho}} \Phi\right)^{2}-\frac{\Delta}{a_{\rho}^{2 / \beta}}\right]=0, \\
& \nabla_{\vec{\rho}}^{2} \Phi-\frac{2 m\left(\vec{\nabla}_{\vec{\rho}} m\right) \cdot\left(\vec{\nabla}_{\vec{\rho}} \Phi\right)}{1-m^{2}}=0,
\end{aligned}
$$

whose solutions must obey the auxiliary conditions $m(0)=$ $m(2 \pi \beta)$ and $\Phi(0)=\Phi(2 \pi \beta)$ due to the global curvature effects. This global effect, i.e., the identification of opposite points of the wedge is fundamental for the existence of vortices in this geometry. With these conditions, one can see that Hamiltonian (3) and the equations of motion (5) and (6) now have all necessary ingredients to produce a vortex solution. A static vortex (for a while assumed to be centralized at the conical apex) is the pair of functions $(m, \Phi)=(f(\rho \sqrt{\Delta}), Q g(\vec{\rho}))$ that satisfy Eqs. (5) and (6). Here $Q= \pm 1, \pm 2, \ldots$ is the topological charge, positive for vortices and negative for antivortices. Away from the vortex center the field $g(\vec{\rho})$ is proportional to the angle $\tau$ and has the form $g=\tau$ (or $\Phi=Q \tau$ ). The field $m(\rho)$ must regularize the vortex at the core, but it has a strong dependence on the anisotropy, $\delta$, and on the cone aperture, $\beta$, as well. In the usual flat space, this field can be obtained only numerically and the analysis leads to two different types of vortices known as in-plane and out-of-plane vortices, depending on whether the static vortex has zero or nonzero out-of-plane spin components [14], respectively. In this case, the out-of-plane region is restricted to a small core region (of size $l_{0}=a / \sqrt{\delta}$ ) at the vortex center. Here, in contrast to the standard flat space case, spins placed at sites with different values of $\rho$ experience different anisotropies. We should stress that such a feature is not characteristic of the material itself, once $\delta$ was assumed to be the same at all sites, rather it is related to the underlying geometry of the magnet. Indeed, for any $\delta$ and $\beta \neq 1$, the effective anisotropy vanishes in the limit $\rho \rightarrow 0$, and therefore, the spin system behaves like an (almost) isotropic magnetic material around the conical apex. Hence, independently of the values of $\delta$, "out-ofcone" spin components could also develop around the tip of the cone. On the other hand, as $\rho$ increases, the effective anisotropy 
grows considerably so that the spins are expected to be confined at the conic surface. Thus, out-of-cone fluctuations are not expected to be observed far away from the tip.

Nevertheless, based on the above discussion and on the results for soliton configurations on the conical surface (see Ref. [7]), we also expect two types of vortex solutions, which will be referred to as 'in-cone' and 'out-of-cone' vortices. Of course, it would be energetically favorable for out-of-cone vortices to nucleate preferentially around the cone apex, while, in contrast, in-cone vortices would prefer to nucleate away from this region. For the particular case of a vortex centered at the cone apex, results of Ref. [7], concerning the problem of an isotropic magnetic system on a cone, suggest that $m(0)= \pm 1$, while the term $\Delta=\delta \beta^{-2} \rho^{\frac{2-2 \beta}{\beta}}$ should imply $m(\rho) \rightarrow 0$ for $\rho \rightarrow \infty$. Really, the term $\int\left(\Delta m^{2}\right) \rho d \rho$ in Hamiltonian (3) diverges if $m \neq 0$ at $\rho \rightarrow \infty$. Consequently, the configuration of an out-of-cone vortex, which may nucleate only around the cone tip, can be summarized as follows: $\Phi=Q \tau, m(0)=p$, $m(\rho) \rightarrow 0$ for $\rho \rightarrow \infty$, where $p= \pm 1$ is the so-called polarity of the vortex. The size of the out-of-cone region can be estimated, in analogy to $l_{0}=a / \sqrt{\delta}$, if we take $l_{\rho}(\beta, \delta)=$ $e^{\beta} a_{\rho} \beta^{\beta /(1-\beta)} / \sqrt{\delta}$. For a fixed $\delta, l_{\rho}$ grows considerably whenever $\beta$ is decreased. Then, out-of-cone magnetic interactions play important roles as the conical apex becomes sharper. However, it is reasonable to assume that such interactions can be neglected when the surface is nearly planar and/or the values of $\delta$ are sufficiently large. In contrast to "out-of-cone" vortices, the "in-cone" vortices, characterized by a core with spins confined at the conical surface, have more chance of nucleating away from the cone tip, i.e., their centers may not be found at $\rho=0$ (unless, of course, $\delta \gg 0$ and/or $\beta \approx 1$ ). Besides, it is also interesting to note that such excitations can have relatively strong out-of-cone spin fluctuations around the conical apex.

Here, we shall firstly specialize to cylindrically symmetric solutions on the surface of a circular cone, say, $m=m(\rho)$ and $\Phi=\Phi(\tau)$ with unity topological charge, $Q= \pm 1$. In this case, asymptotic solutions for out-of-cone vortices can be obtained. The region defined by $\rho<l_{\rho}(\beta, \delta)$ is nearly isotropic and the $\Delta$-term can be neglected. Hence, based on results of Ref. [7] one can easily find the out-of-cone vortex configuration for $\rho \ll$ $l_{\rho}(\beta, \delta)$

$m_{\mathrm{oc}} \cong-p\left(1-\frac{\rho^{2}}{l_{\rho}^{2}}\right), \quad \Phi_{\mathrm{oc}}=\tau$.

For the outer region $\rho \gg l_{\rho}(\beta, \delta)$, one has $m_{\mathrm{oc}}=0$ and $\Phi_{\mathrm{oc}}=\tau$. The exchange energy of such a solution may be estimated, using Eq. (4), to be:

$$
\begin{aligned}
E_{\mathrm{oc}} \approx & \pi J \beta \ln \left(\frac{L}{l_{c}}\right) \\
& +2 \pi \beta J\left[\frac{3}{4}+2 \ln (2)+\frac{\beta \delta}{(\beta+1)(2 \beta+1)} \frac{l_{c}^{2}}{a^{2}}\right],
\end{aligned}
$$

where $L$ and $l_{c}\left(l_{c}=\left(\beta l_{\rho}\right)^{1 / \beta}=\left(e a \beta^{1 /(1-\beta)} / \delta^{1 / 2 \beta}\right)\right)$ represent the cone size and the out-of-cone core region size, respectively (in units of the actual lattice spacing, $a$ ). Clearly, in the limit of an infinite cone, the first term blows up; the second one, taking into account the contribution from the core, is finite. Notice also that, the cone geometry plays an important role in the energy behavior, so that it linearly decreases with conical aperture, vanishing in the limit $\beta \rightarrow 0$, even if $L \rightarrow \infty$. For arbitrary $Q$ energy above is proportional to $Q^{2}$.

On the other hand, whenever $l_{c}(\beta, \delta) \rightarrow 0$ (strong anisotropy and $\beta$ large enough), in-cone vortex solutions given by $\left(m_{i c}=0\right.$, $\left.\Phi_{i c}=\tau\right)$ must become stable, even at the tip. In this case, the in-cone vortex energy reads

$E_{\text {ic }}=\pi J Q^{2} \beta \ln \left(L / a_{0}\right)$,

where $a_{0}$ is a cutoff introduced to prevent spurious divergences associated to the continuum approach and also to provide a correct value for the exchange energy. For example, for an in-plane vortex on a planar square lattice we have that $a_{0} \approx 0.24 a$ [13]. Similarly to the out-of-cone solution, also here energy linearly decreases with $\beta$-parameter, while diverging like $\ln (L)$. A detailed study of how the field $m$ depends on the vortex position and how vortex stability depends on the anisotropy and curvature is more involved and we plan to do this in the near future. In addition, note that since the energies (8) and (9) differ by a finite term, then results obtained for one kind of vortex could be, at some extent, applied to the another.

The main conclusion is the following: whenever lying on the surface of a cone, a vortex-like excitation presents lower exchange energy than its flat plane counterpart. Moreover, as cone is narrowed, such an energy linearly decrease. We may understand this in a simple geometric way, like follows: a cone with a narrower opening angle corresponds to a smaller magnet, which therefore has less magnetic energy.

It should be noticed that Belavin-Polyakov-like solitons present a similar characteristic whenever defined on a conical support [7], say, their energy also linearly decreases with $\beta$ parameter (see, however, Ref. [8], where is considered another solution, on a truncated cone, whose energy does not depend on the conical aperture angle). Furthermore, we shall see, in the next section, that vortex energy is lower whenever it nucleate around conical apex, a fact that could be interpreted as a geometrical pinning of magnetic vortex.

\section{The geometrical pinning of magnetic vortices on a conical support}

In the preceding section, our analysis was performed for a vortex centered on the conical apex, where we obtained the energies of the two possible solutions. We may wonder whether these are the unique possible cases. Actually, as we shall show below these are the most energetically favorable configurations but not the unique ones. As we have seen, outside the apex region spins tend to be encountered lying on the surface. Therefore, we shall explicitly deal hereafter with the in-cone excitations. In order to see this, let us consider an in-cone magnetic vortex whose center is apart $D$ from that point which, after identification of lines, $\mathbf{u}=\mathbf{v}$, will play the role of the conical apex. Furthermore, it should be emphasized that the cylindrical symmetry of the vortex must be verified accord- 

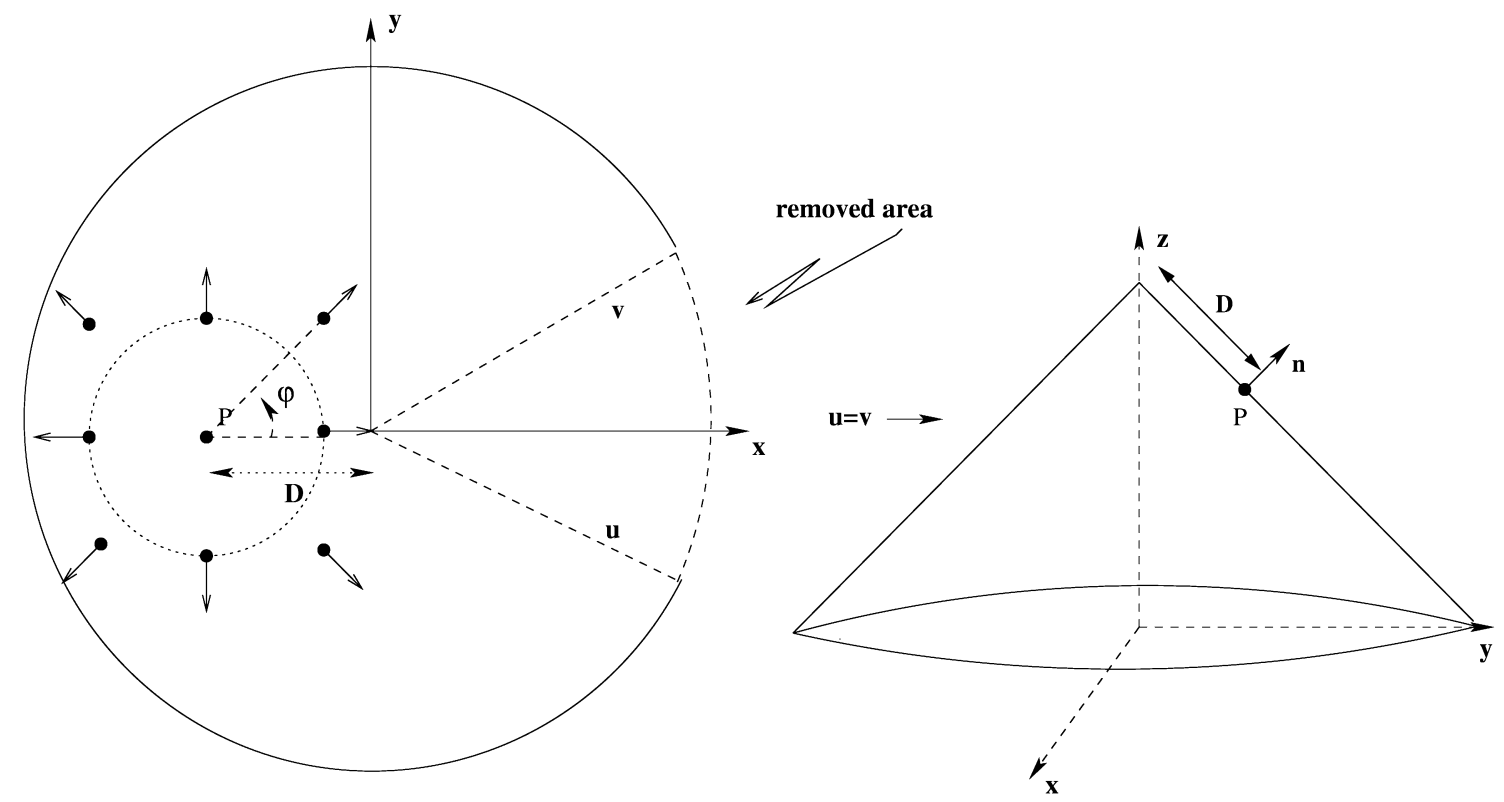

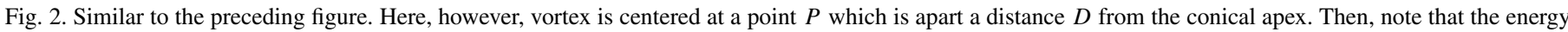

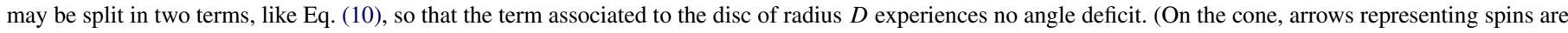
not depicted to improve visualization.)

ing to $\mathbf{n}$-vector. Such a configuration centralized at the point $\left(x_{0}, y_{0}\right)$ on the plane with a wedge, reads (up to a constant), $\Phi_{D}=\arctan \left[\left(y-y_{0}\right) /\left(x-x_{0}\right)\right]$, whose energy may be explicitly calculated to be

$$
\begin{aligned}
E(D, \beta) & =Q^{2} \int_{a_{0}}^{D+a_{0}} \int_{0}^{2 \pi} \frac{d r}{r} d \varphi+\int_{D+a_{0}}^{L} \int_{0}^{2 \pi \beta} \frac{d r}{r} d \varphi \\
& =\pi Q^{2} J \ln \left[\frac{L^{\beta}\left(D+a_{0}\right)^{(1-\beta)}}{a_{0}}\right],
\end{aligned}
$$

where $\varphi$ measures the relative angle of the spins relative to an axis joining the point $\vec{P}$ and the origin. Essentially, as illustrated in Fig. 2, all the spins inside a disc of radius $D$, centered at $P$, are kept after identification of lines, so that no angle deficit effect is experienced by this term. However, a number of the outer sites should be removed from energy considerations, what gives rise to the factor $2 \pi \beta$ in the angular integration of the second term, above. (The vortex core radius, $a_{0}$, is introduced to avoid spurious divergence in the calculation.)

Some limits are in order: first, note that as long as $\beta \rightarrow 1$ then $E(D, \beta=1)=\pi J \ln \left(L / a_{0}\right)$ recovering its flat counterpart. On the other hand, whenever $\beta \rightarrow 0$ then $D$ is meaningless and vanishes, so that $E(\beta=0)=0$. Now, if we set $D=0$ we recover equation (9), as expected. However, as long as $D$ becomes very large, $D \rightarrow L \rightarrow \infty$, vortex energy is unaffected by conical geometry, say, $E(D \rightarrow \infty, \beta)=\pi J \ln \left(L / a_{0}\right)$. The last result may be easily understood if we remember that the vortex energy density is proportional to $r^{-2}$, so that, quite apart from the vortex core, it is very small and so practically not sensitive to area deficit. This is also the reason why its total energy is increased as long as its center is moved away from the conical apex (Fig. 3).

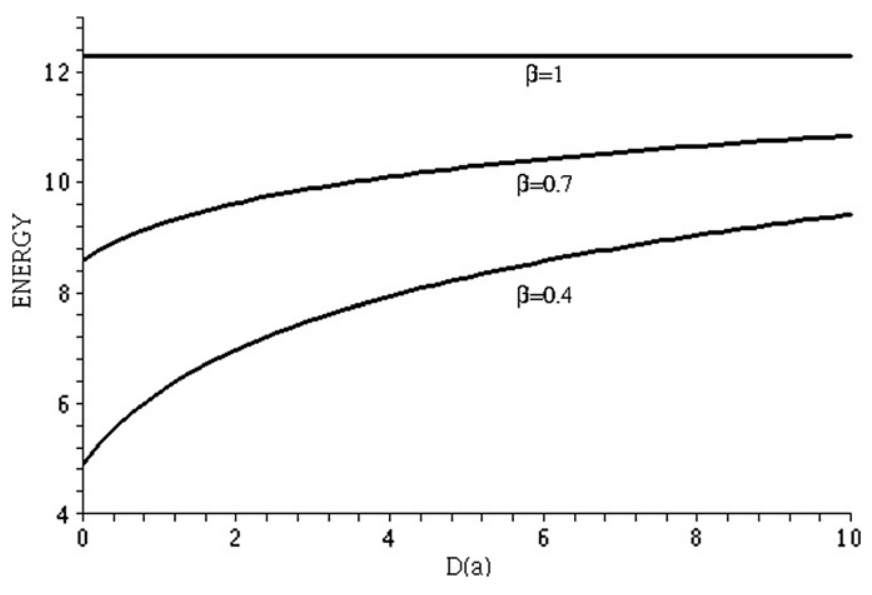

Fig. 3. The energy of a single vortex on a cone as function of its center distance from the tip, $D(a)$. We have taken $J, Q$ and $a_{0}$ equal to the unity while $L=50 a_{0}$.

Thus, we may understand that apex attracts magnetic in-cone vortex according to

$$
\begin{aligned}
V_{\text {apex }}(D, \beta) & =E(D, \beta)-E_{\mathrm{ic}} \\
& =\pi Q^{2} J(1-\beta) \ln \left[1+\frac{D}{a_{0}}\right],
\end{aligned}
$$

so that in the limit $D / a_{0} \ll 1$ we get a linearly confining potential, $\pi J(1-\beta) D / a_{0}$. It should be emphasized that, although $a_{0}$ is the smallest cutoff length scale of the model, the limit $D / a_{0} \ll 1$ simply states that whenever the vortex is pinned to the apex cone, a strong attractive potential appears against dislocations of its center from that point (Fig. 4). This is to say that, if the vortex center could develop small oscillations around the apex, it would experience the linear confining potential presented above. For the sake of comparison, a similar small 


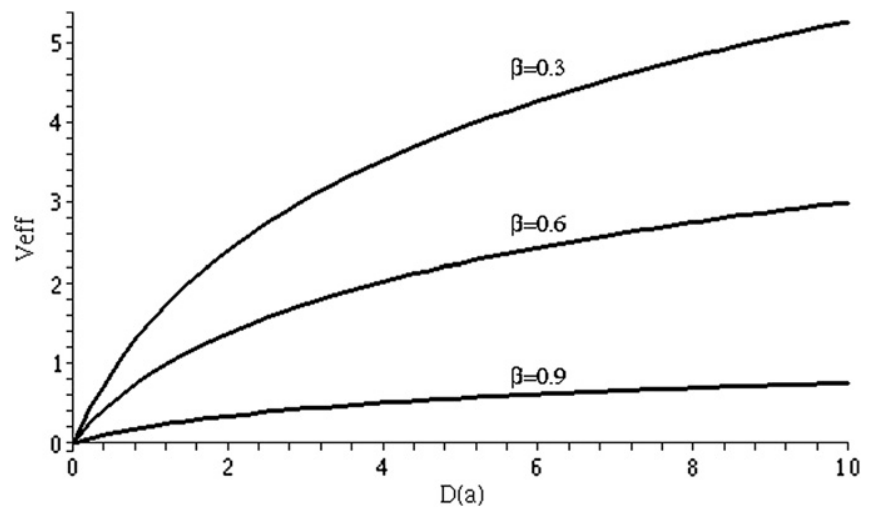

Fig. 4. The effective potential of the apex on a in-cone vortex as function of its center distance from the tip, $D(a)$. We have taken $J, Q$ and $a_{0}$ equal to the unity while $L=50 a_{0}$.

oscillation of a vortex around spin vacancies was predicted for diluted easy-plane magnets [15]. Here, such a scenario resembles quark confinement, where a linear potential provided by gluons keeps quarks together. In addition, in the limit $\beta \rightarrow 0$ such a potential is not continuous since a naive calculation gives $V_{\text {apex }}(D, \beta=0)=\pi J \ln \left(1+D / a_{0}\right) \neq 0$. However, as stated above, $D=0$ as long as $\beta=0$, so that this potential must vanish in this case. Therefore, expression (11) is explicitly valid only for non-degenerate cone, say, $0<\beta \leqslant 1$. Such a defect in the space may also cause important effects in the spin dynamics and configurations. Really, it is expected that the spins in the neighborhood of the cone apex, which is a singularity, must have larger fluctuations than the distant spins. Then, it is conceivable that an in-cone vortex reaching the tip should change to an out-of-cone excitation, unless $\delta$ be strong enough to keep them on the cone surface. Furthermore, the translational symmetry is broken on this surface since there is a preferential position for the nucleation of these excitations.

Because apex nucleated magnetic vortices appear to be stable states, we may wonder whether this could be thought as a mechanism for magnetic recording and logic gates, for example, in the lines put forward in Refs. $[9,10,16]$. Furthermore, following ideas of Ref. [16], we may wonder whether conical shaped defects could not be incorporated into submicron magnetic particles so that vortices would appear nucleated around conical spices instead of hole-pinned vortices metastable states. Besides, there is a similarity between the system studied here and magnetic force microscope tips, where it is well known that a highly pointed tip implies in localization of a strong magnetic field there. Also in this line, we could combine tips and nonmagnetic impurities, which has also been shown to attract topological objects [17-25]. Then, a scenario with more than one kind of attracting point may give rise to externally controllable multistable states of vortices nucleated around tips or impurities.

\section{Two vortices on the cone}

At some extent, a vortex is similar to an elementary charge in electrostatics, even in a conical surface. Indeed, the self-energy of both objects blows up at both $\rho \rightarrow 0$ and $\rho \rightarrow \infty$ limits, like in the usual flat plane system. Actually, the singularity at $\rho=0$ may be regularized by an additional short-range field $(m(\rho))$ to form a finite vortex core, but for a strong enough anisotropy, the spins tend to lie on the surface even in the region of the vortex core (around a plaquette). However, the logarithmic divergence for large $\rho$ remains so that a single vortex energy blows up in an infinite system (here, an infinite cone). Therefore, other vortices must be present. For instance, if a vortex appears together with an antivortex (to form the equivalent of a dipole) then the pair energy is finite. Thus, a natural question to be answered is how vortices interact on the conical surface. For that, let us consider the case of two vortices, with charges $Q_{1}$ and $Q_{2}$, on the cone. Here, we shall assume that $\delta \gg 1$, so that only in-cone vortices are expected to be found (see Section 2). (The scenario with transition to out-of-cone profiles very close to the apex could be considered, at principle, in the same lines.) We use $x-y$ coordinates of a plane with a deficit angle (we take the origin to be at conical apex). Their profile reads like follows:

$\Phi_{Q_{1} Q_{2}}=Q_{1} \arctan \left(\frac{y-y_{v}}{x-x_{v}}\right)+Q_{2} \arctan \left(\frac{y-y_{\tilde{v}}}{x-x_{\tilde{v}}}\right)$,

whose energy may be analytically carried out and reads:

$$
\begin{aligned}
& E_{Q_{1} Q_{2}}(D, \beta) \\
& \quad=\pi^{2} \beta J\left(Q_{1}^{2}+Q_{2}^{2}\right) / 2+\pi \beta J\left(Q_{1}+Q_{2}\right)^{2} \ln (L / a)+V_{\text {eff }} .
\end{aligned}
$$

Here, the first term is the energy formation of vortices while the second answers for their energies according to the sample size, $L$. In addition, the effective potential, $V_{\text {eff }}$, is given by

$$
\begin{aligned}
V_{\mathrm{eff}}( & \left.Q_{i}, D, \beta\right) \\
= & -2 \pi \beta J Q_{1} Q_{2} \ln (\mathcal{D} / a) \\
& +\sum_{i} \pi J(1-\beta) Q_{i}^{2} \ln \left(1+\frac{\left|\vec{D}_{i}\right|}{a_{0}}\right),
\end{aligned}
$$

where $\vec{D}_{i}(i=1,2)$ localize the vortices separated by $\mathcal{D}$ (the less path joining the two vortices, generally appearing to be an arc segment on the cone). (Note that, as long as $\beta \rightarrow 1$ usual plane-like results are recovered.) Clearly, the first term in Eq. (14) appears to be repulsive (attractive) if the vortices present charges of the same (opposite) signals, like usually (but depending on $\beta$-parameter). Thus, a vortex and an antivortex attract each other while two vortices (or antivortices) mutually repeal. The second term is traced back to the attractive effect of the conical tip on a vortex (or antivortex). Therefore, we have two cases depending upon the signals of the topological charges.

First, if vortices have opposite charges, then they are expected to be attracted to the apex. This fact is illustrated in Fig. 5 which displays how energy of a vortex-antivortex pair with unity charges, $Q_{1}=-Q_{2}=+1$, behaves as function of distance vortex-apex, $D(a)$, for some values of $\beta$ parameter (the antivortex is $\mathcal{D}=a$ apart from the vortex, $Q_{1}$ ). Notice that as long as cone is narrowed the pair energy appears to get lower values. In addition, note also that the potential well provided 


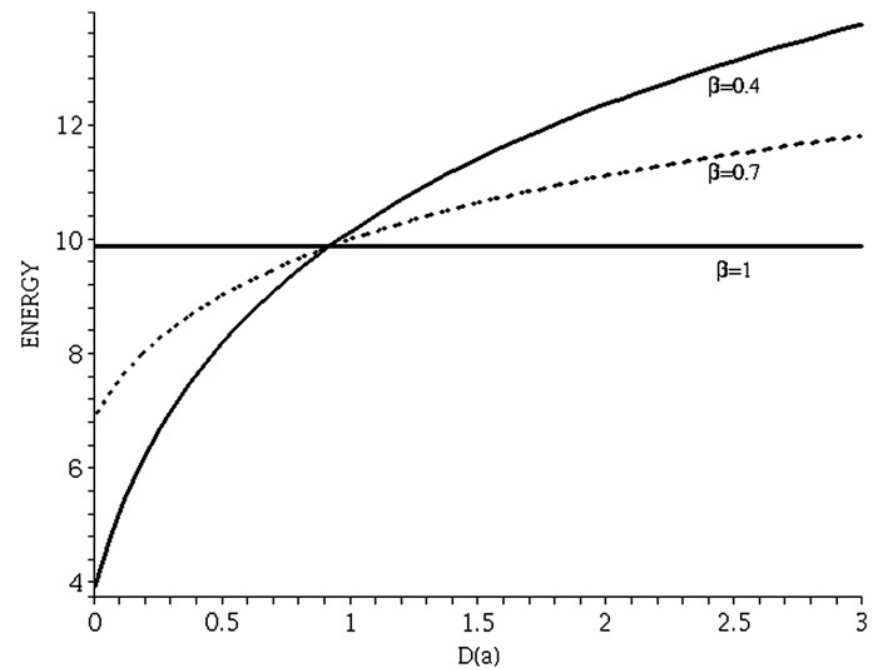

Fig. 5. Shows how the vortex-antivortex pair energy behaves as function of $D(a)$. Clearly, the pair is expected to nucleate around conical apex, $D=0$ (we have taken $\mathcal{D}=a, J=1$.)

by the apex gets deeper, say, the stable states for the pair correspond to one of the vortices pinned to the tip. Analogously to the single vortex case, a geometrical pinning of magnetic vortex is also observed here.

On the other hand, whenever both vortices carry the same signal charges, for simplicity, we take $Q_{1}=Q_{2}=+1$, then $V_{\text {eff }}$, (14), consist of competing potentials: vortices repealing each other while the apex working to keep them together, near the tip. Actually, on the conical surface the apex attraction can dominate mutual repulsion so that a system with two vortices with the same charge may, at principle, nucleate around the tip. In order to investigate such a possibility in more details, let us fix some configurations for the vortices on the cone. First, let us take both of the two vortices at the same distance from the apex, $\left|\vec{D}_{1}\right|=\left|\vec{D}_{2}\right| \equiv D$, but centered at diametrically opposite points (thus, separated by $\mathcal{D}=2 D$ if $\beta>2 / \pi$ or $\mathcal{D}=\beta \pi D$ whenever $\beta \leqslant 2 / \pi)$. For this configuration, the minimum of $V_{\text {eff }}$, Eq. (14), will occur, for a given $\beta$, at

$D_{\min _{1}}=\frac{a_{0} \beta}{(1-2 \beta)}$,

which states that a finite value take place only for $\beta<1 / 2$ (cone aperture half-angle $\alpha<30^{\circ}$ ). In words, given a $\beta<1 / 2$ there exists a distance from the apex, $D_{\min _{1}}$, which supports a stable state of the two vortices bounded to the apex.

Indeed, for $\beta \lesssim 1 /\left(2+a_{0}\right) \approx 0.447 \equiv \beta_{\mathrm{cr}_{1}}\left(\alpha \lesssim 26.55^{\circ}\right)$ stability demands $D<a$. (From the result above for $\beta_{\mathrm{cr}_{1}}$ we clearly realize that vortices-apex nucleation possibility is also intimately related to the finite size of the vortex core, $a_{0}$.) In this case, we would have effectively both vortices at the tip (in the discrete scenario). Energetics could, in this case, favors the formation of an unique $Q=2$ excitation instead of two $Q=1$ vortices. In this line, we should mention the work of Ref. [21], in which doubled charged vortices appear nucleated around spin vacancies in the standard plane, as demonstrated by simulations, by virtue of the attractive effect of such impurities. (Although our results cannot be extrapolated to short range, say

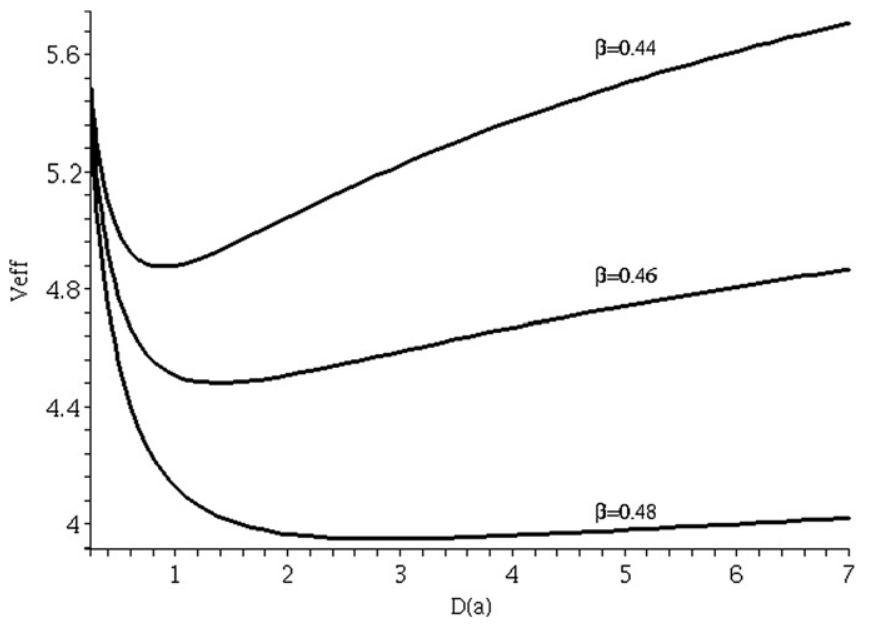

Fig. 6. Shows how $V_{\text {eff }} / J$ behaves as function of $D(a)$ for the case $Q_{1}=Q_{2}=+1$. Note that, in despite of the vortices have the same charge, energetics tends to keep them tied near the conical tip. (The values for $D_{\min _{1}}$ read around $0.88 a, 1.38 a$ and $2.88 a$ for $\beta$ equals to $0.44,0.46$ and 0.48 , respectively).

$D \lesssim a$, it would be interesting to simulate a similar system on the conical surface.) On the other hand, whenever $\beta \gtrsim 0.447$, the two vortices nucleate around the tip with $D \geqq a$. This could be viewed as a vortices-tip bound state, since the effective potential presents a global minimum at $D_{\min _{1}}$. Let us recall that such a possibility is ruled out from usual planar-like systems. Fig. 6 displays how the effective potential behaves as function of $D(a)$ for some values of $\beta$. As may be clearly realized, as long as $\beta$ increases from $\beta_{\mathrm{cr}_{1}}$ to $1 / 2$ then $D_{\min _{1}}$ shifts to higher values.

Another possible configuration is that in which one vortex is already centered at the apex while the another is apart $D$. In this case, one of the terms presented in the sum of Eq. (14) identically vanishes. The remaining potential is minimized at

$D_{\min _{2}}=\frac{2 a_{0} \beta}{(1-3 \beta)}$.

Now, stability is possible only for narrower cones, say $\beta<$ $1 / 3\left(\alpha \lesssim 19.47^{\circ}\right)$. However, according to the relation above, vortices-apex stable states with $D \geqslant a$ demands $\beta \lesssim 0.287 \equiv$ $\beta_{\mathrm{cr}_{2}}$. In summary, we conclude that the nucleation of two vortices (or antivortices, as well) around the conical tip is possible for some narrow apertures. If the two objects are separated from the apex of $D=D_{\min _{1}} \geqslant a$, then cone aperture half-angle $\alpha \equiv \alpha_{1} \in\left(26.55^{\circ}, 30^{\circ}\right)$. However, if one object is already nucleated at the tip and the another is $D=D_{\min _{2}} \geqslant a$ apart, a narrower cone is demanded, say $\alpha_{2} \in\left(16.68^{\circ}, 19.47^{\circ}\right)$, approximately.

Let us now consider the problem of vortex-pair dissociation in this background. Our estimative will consider only in-cone vortices. First of all, let us emphasize that it is nowadays wellestablished that the critical temperature, of vortex-antivortex depairing, is $\approx 0.284 \pi J / k_{B}$ [26], within the planar rotator, while for the $X Y$-model, where spins can have out-of-plane fluctuations, it reads $\approx 0.22 \pi J / k_{B}$ (see Ref. [27] for details). However, it has been recently raised the question of how vacan- 
cies affect this transition (see, for example, [20,21,23,28] and related references therein). Actually, several simulations have shown that vacancies tend to diminish the transition temperature, so that at vacancy concentrations $\varrho$ equal or above the percolation threshold $\varrho_{p t} \approx 0.41$, the topological transition is ruled out at any finite temperature [23].

As we have noted in the introduction, the deficit angle can be viewed as a region with nonmagnetic impurities grouped in a cluster with a wedge shape (see Fig. 1). In this case, the impurity concentration would be proportional to $2 \pi \gamma$, i.e., proportional to the deficit angle (the problem with this interpretation is the boundary conditions required at the edges of the wedge). To estimate the critical temperature for the conical surface we use the same argument of energy-entropy as Kosterlitz-Thouless performed for the standard plane [2]. Here, the system area is given by $\beta L^{2}$ and then, the entropy may be estimated to be ${ }^{1}$ $S_{\text {cone }}=k_{B} \ln \left(\beta L^{2} / \beta a^{2}\right)=2 k_{B} \ln (L / a)$, like in the usual planar case. Therefore, a rough analysis gives the following range for depairing temperature of vortex-antivortex excitations in the $X Y$ model defined on a conical geometry

$\pi \beta J<k_{B} T_{\text {cr-cone }}<\pi J$.

Actually, if we could extrapolate results of Ref. [27] to the present geometry, we would get $0.22 \pi \beta J<k_{B} T_{\text {cr-cone }}<$ $0.22 \pi J$. Therefore, the critical temperature $T_{c}$ of the system have a linear dependence on the geometrical parameter $\beta$.

\section{Conclusions and prospects}

In the present work we have considered vortex-like excitations of $X Y$-like model on a circular conical support. Such a space is locally Euclidean, since a conical singularity is created after removing a wedge of the usual plane and gluing its edges. Vortex solutions and configurations depend on vortex position and deficit angle in a nontrivial way so that our analysis of this problem has not been rigorous. Nevertheless, the results presented here may be of interest for many condensed matter systems (2D electrons gas on the cone) and field theories on manifolds with nontrivial geometry as well as for lower dimensional gravity, in which the anisotropic nonlinear $\sigma$ model and its associated vortex-like solutions are coupled to the Einstein field. We have seen that whenever lying on a cone, vortex (or antivortex) energy appears to linearly decrease with conical aperture angle, so that as cone is extremely tightened $(\beta \rightarrow 0)$, its energy vanishes like sample size does. We have studied incone and out-of-cone vortex excitations. For suitable ranges of the anisotropy, the first type appear to be found far away from the tip while the second ones tend to be centered at the apex. In addition, we have seen that it is energetically favorable for a magnetic vortex to nucleate around the conical apex. Thus, an in-cone could become an out-of-cone vortex as long as it is

\footnotetext{
1 Since the apex is more energetically favorable than other points, we have a nonuniform probability distribution over the surface. Thus, our estimative is good if a small area around the tip is not considered. Therefore, our analysis is strictly valid only for in-cone excitations, whose centers are expected to be moved away from the apex.
}

trapped by the tip. Therefore, we could think conic-like magnets as pinning defects for such excitations.

Going further, we have taken two vortices on the cone. Analogously to the former case, even though its energy has a logarithmic dependency on their distances, it also depends linearly on $\beta$ parameter. In the case of a vortex-antivortex pair $\left(Q_{1}=-Q_{2}=+1\right)$, our results show that it is energetically favorable for nucleation around the apex, like in the single vortex case. An intriguing result emerges as long as we take $Q_{1}=Q_{2}$. Although repealing each to other, like in the usual planar-like case, conical apex tends to attract and keep them around the tip. Actually, we have realized that for conical aperture smaller than a critical value, apex potential dominates repulsion, which leads to the nucleation of the vortices. As a final result, connecting geometrical aspects with Berezinskii-Kosterlitz-Thouless transition related issues, on a cone, such a transition is expected to takes place at lower temperatures.

As prospects for future investigation we may quote, for example: (i) simulations of spin models in order to confirm the assertions and results presented here; (ii) study the "out-of-cone vortices" and possible other deformed solution such as spiral vortices [29], which could be present in non-circular cones; (iii) consider the spin dynamic in curved spaces and/or in surfaces containing defects [15]; (iv) study of magnetostatic energy on the cone surface, mainly in connection with nanosized magnets.

Finally, our present study could be also useful for some related systems, like superconducting vortices, dislocations, and so forth, whenever they lie in conic-like geometries.

\section{Acknowledgements}

The authors thank CAPES, CNPq and FAPEMIG for financial support. W.A.M.-M. also thanks DCB/UFVJM where part of this work was done.

\section{References}

[1] V.L. Berezinskii, Sov. Phys. JETP 32 (1970) 493; V.L. Berezinskii, Sov. Phys. JETP 34 (1972) 610.

[2] J.M. Kosterlitz, D.J. Thouless, J. Phys. C 6 (1973) 1181.

[3] R.P. Cowburn, D.K. Koltsov, A.O. Adeyeye, M.E. Welland, D.M. Tricker, Phys. Rev. Lett. 83 (1999) 1042;

T. Shinjo, T. Okuno, R. Hassdorf, K. Shigeto, T. Ono, Science 289 (2000) 930;

T. Pokhil, D. Song, J. Nowak, J. Appl. Phys. 87 (2000) 6319.

[4] See, for example, R.P. Cowburn, M.E. Welland, Science 287 (2000) 1466, and related references therein.

[5] See, for example, A. Saxena, R. Dandoloff, T. Lookman, Physica A 261 (1998) 13.

[6] R. Dandoloff, S. Villain-Guillot, A. Saxena, A.R. Bishop, Phys. Rev. Lett. 74 (1995) 813;

S. Villain-Guillot, R. Dandoloff, A. Saxena, A.R. Bishop, Phys. Rev. B 52 (1995) 6712;

R. Dandoloff, A. Saxena, Eur. Phys. J. B 29 (2002) 265.

[7] A.R. Pereira, J. Magn. Magn. Mater. 285 (2005) 60;

W.A. Freitas, W.A. Moura-Melo, A.R. Pereira, Phys. Lett. A 336 (2005) 412;

See also, V.B. Bezerra, C. Romero, S. Chervon, Int. J. Mod. Phys. D 14 (2005) 1927.

[8] A. Saxena, R. Dandoloff, Phys. Rev. B 66 (2002) 104414. 
[9] C.A. Ross, M. Farhoud, M. Hwang, H.I. Smith, M. Redjdal, F.B. Humphrey, J. Appl. Phys. 89 (2001) 1310.

[10] C.A. Ross, S. Haratani, F.J. Castaño, Y. Hao, M. Hwang, M. Shima, J.Y. Cheng, B. Vögeli, M. Farhoud, M. Walsh, H.I. Smith, J. Appl. Phys. 91 (2002) 6848.

[11] H. Shima, Y. Sakaniwa, J. Phys. A: Math. Gen. 39 (2006) 4921;

H. Shima, Y. Sakaniwa, cond-mat/0512167;

See also, B. Jancovici, G. Téllez, J. Stat. Phys. 91 (1998) 953;

B. Jancovici, G. Téllez, J. Stat. Phys. 116 (2004) 205;

In connection with vortices pinning on surfaces, see, for example, P. Voll, N. Roberts-Warren, R.J. Zieve, cond-mat/0601395;

See also, V. Vitelli, A.M. Turner, Phys. Rev. Lett. 93 (2004) 215301.

[12] J.D. Brown, Lower Dimensional Gravity, World Scientific, New Jersey, 1988;

M. Welling, gr-qc/9511211;

M.O. Katanaev, Phys. Usp. 48 (2005) 675.

[13] G.M. Wysin, Phys. Rev. B 54 (1996) 15156.

[14] G.M. Wysin, Phys. Lett. A 240 (1998) 95.

[15] A.R. Pereira, S.A. Leonel, P.Z. Coura, B.V. Costa, Phys. Rev. B 71 (2005) 014403;

F.M. Paula, A.R. Pereira, G.M. Wysin, Phys. Rev. B 72 (2005) 094425.

[16] M. Rahm, J. Biberger, V. Umansky, D. Weiss, J. Appl. Phys. 93 (2003) 7429;

M. Rahm, J. Stahl, W. Wegsheider, D. Weiss, Appl. Phys. Lett. 85 (2004) 1553.

[17] C.E. Zaspel, C.M. McKennan, S.R. Snaric, Phys. Rev. B 53 (1996) 11317; L.A.S. Mól, A.R. Pereira, W.A. Moura-Melo, Phys. Rev. B 67 (2003) 132403;
G.M. Wysin, Phys. Rev. B 68 (2003) 184411;

F.M. Paula, A.R. Pereira, L.A.S. Mól, Phys. Lett. A 329 (2004) 155

[18] L.A.S. Mól, A.R. Pereira, A.S.T. Pires, Phys. Rev. B 66 (2002) 052415.

[19] A.R. Pereira, A.S.T. Pires, J. Magn. Magn. Mater. 257 (2003) 290.

[20] A.R. Pereira, L.A.S. Mól, S.A. Leonel, P.Z. Coura, B.V. Costa, Phys. Rev. B 68 (2003) 132409.

[21] G.M. Wysin, Phys. Rev. B 71 (2005) 094423.

[22] A.R. Pereira, J. Magn. Magn. Mater. 279 (2004) 396.

[23] G.M. Wysin, A.R. Pereira, I.A. Marques, S.A. Leonel, P.Z. Coura, Phys. Rev. B 72 (2005) 094418.

[24] K. Subbaraman, C.E. Zaspel, J.E. Drumheller, Phys. Rev. Lett. 80 (1998) 2201;

M.-H. Julien, T. Fehér, M. Horvatić, C. Berthier, O.N. Bakharev, P. Ségransan, J.-F. Marucco, Phys. Rev. Lett. 84 (2000) 3422;

J. Bobroff, H. Alloul, W.A. MacFarlane, P. Mendels, N. Blanchard, G. Collin, J.-F. Marucco, Phys. Rev. Lett. 86 (2001) 4116.

[25] A.R. Pereira, Phys. Rev. B 71 (2005) 224404; A.R. Pereira, J. Appl. Phys. 97 (2005) 094303.

[26] P. Olsson, Phys. Rev. Lett. 73 (1994) 3339; P. Olsson, Phys. Rev. B 52 (1995) 4526.

[27] A. Cuccoli, V. Tognetti, R. Vaia, Phys. Rev. B 52 (1995) 10221; H.G. Evertz, D.P. Landau, Phys. Rev. B 54 (1996) 12302.

[28] S.A. Leonel, P.Z. Coura, A.R. Pereira, L.A.S. Mól, B.V. Costa, Phys. Rev. B 67 (2003) 104426.

[29] A.B. Borisov, I.G. Bostrem, A.S. Ovchinnikov, Phys. Rev. B 72 (2005) 134423. 